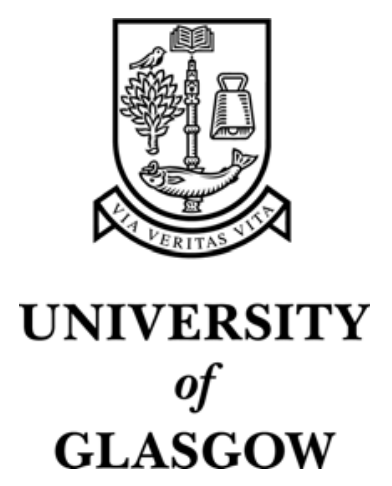

Johnston, S. F. (2003) An unconvincing transformation? Michelson's interferential spectroscopy. Nuncius:annali di storia della scienza 18(2):pp. 803-823.

http://eprints.gla.ac.uk/archive/2893/ 


\title{
AN UNCONVINCING TRANSFORMATION? MICHELSON'S INTERFERENTIAL SPECTROSCOPY
}

\author{
Sean F. Johnston*
}

\section{Introduction}

Albert Abraham Michelson (1852-1931), the American optical physicist best known for his precise determination of the velocity of light and for his experiments concerning aether drift, is less often acknowledged as the creator of new spectroscopic instrumentation and new spectroscopies. His researches on the velocity of light (1878-9) and aether drift $(1881-7)^{1}$ exploited his excellence in precision optical measurement and metrology. They were closely followed by work on standards of length (188793) and the diameters of stars (1890-1). The measurement of the standard meter brought Michelson to the exploration of spectroscopy. He devised a new method of light analysis relying upon his favourite instrument - a particular configuration of optical interferometer - and published investigations of spectral line separation, Doppler-broadening and simple high-resolution spectra (1887-1898). Contemporaries did not pursue his method. Michelson himself discarded the technique by the end of the decade, promoting a new device, the 'echelon spectroscope', as a superior instrument. High-resolution spectroscopy was taken up by others at the turn of the century using the echelon, Fabry-Pérot etalon and similar instruments. Michelson's 'Light Wave Analysis' was largely forgotten, but was 'rediscovered' c1950 and developed over the following three decades into a technique rechristened 'Fourier transform spectroscopy'. ${ }^{2}$

This paper presents Michelson's interferometric work as a continuum of personal interests and historical context. Richard Staley has noted how Michelson's career is too often sketched as a few key episodes; instead, he cites Michelson as a prototypical 'academic mechanician' bridging science and engineering via a programme of instrumental innovation. ${ }^{3}$ A more inclusive categorisation that applies beyond the American context is that of 'research-technology'. ${ }^{4}$ Indeed, Michelson's career trajectory, and others in optics, can be described even more generically as 'peripheral sciences' having distinct occupational, professional and intellectual characteristics. ${ }^{5}$

* University of Glasgow Crichton Campus, Dumfries DG1 4ZL, UK, s.johnston@crichton.gla.ac.uk

${ }^{1}$ But, indeed, pursued nearly until the year of his death, as were his velocity of light experiments; see L. S. SWENSON JR, The Ethereal Aether: A History of the Michelson-Morley-Miller Aether-Drift Experiments, 18801930 (Austin: University of Texas Press, 1972).

2 'Interferential spectroscopy' is a conjunction apparently never used by Michelson himself. He referred to his techniques by a variety of names, opting for 'the method of light-wave analysis' in later works. However, Michelson generally employed the adjective 'interferential' in preference to 'interferometric', so this term has been employed throughout to provide clarity. 'Interference spectroscopy' (e.g., in R. W. Wood, Physical Optics (New York: MacMillan, 1923)) or 'interferential spectroscopy' were terms popularised during the early 20th century. Fourier transform spectroscopy (FTS), coined in the mid 1950s, has also been known as Fourier spectroscopy, interferometric spectroscopy, or Fourier Transform Infrared (FTIR) spectroscopy.

${ }^{3}$ R. STALEY, "Travelling Light," in Natural Standards (Max-Planck-Institut fur Wissenschaftsgeschichte, 2001), 37-65.

${ }^{4}$ B. Joerges and T. Shinn, eds., Instrumentation: Between Science, State and Industry (Dordrecht: Kluwer, 2000).

${ }^{5}$ S. F. Johnston, A History of Light and Colour Measurement: Science in the Shadows (Bristol: Institute of Physics Publishing, 2001). 
While being firmly situated in a wider, if too often unrecognised, research tradition, Michelson was also a transitional figure. His interferential methods proved unpalatable to visually centred spectroscopists of the late nineteenth century, but were typical of the increasingly quantitative and analytical methods that became popular between the world wars. As such, his work provides a convenient bridge between the historiographies of nineteenth and twentieth century optics. This paper examines the sources and content of Michelson's new experimental knowledge, and explores the social and technical reasons for the muted reception to his methods. Before tackling the events themselves, however, it is necessary to peer through the layers of historical interpretation that have accreted over the past century.

\section{Historiography}

Michelson's spectroscopic work had a highly variable reception that was dependent on changing scientific fashion and technical cultures. During his lifetime, the only extensive writings on the subject were by Michelson himself. Michelson first published on the use of monochromatic light (or, as he termed it, light from an 'approximately homogeneous source') as a standard of length in the late 1880s with Edward W. Morley, shortly after their famous aether drift experiment. ${ }^{6}$ Within four years he began to publish explicitly on spectroscopic connections. ${ }^{7}$ This work was summarised in the popular lectures Light Waves and Their Uses, in a review paper and, much later with minor revisions, in his Studies in Optics. ${ }^{8}$ The importance of this body of work to contemporary physics is suggested by his 1907 Nobel prize 'for his optical precision instruments and the spectroscopic and metrological investigations carried out with their aid'.

Michelson's spectroscopic researches were nevertheless described with brevity by subsequent commentators. There are few contemporaneous papers on the subject. One contemporary textbook, for which Michelson tellingly provided the preface to the English edition, devoted favourable attention to all his research, with five pages allocated to interferential spectroscopy. ${ }^{9}$ Some 15 years later, related research was published with, however, little or no reference to Michelson. ${ }^{10}$ By the interwar period, the method was generally judged to have been a failed, or at best transitional, technique; typical optics texts described all Michelson's research except interferential spectroscopy or rather dismissively. ${ }^{11}$ Texts from the late 1950s, however, began once again to devote space to Michelson's spectroscopic research, owing to the rehabilitation of some aspects of the method in the form of Fourier transform spectroscopy (FTS). ${ }^{12}$ Concepts of Classical Optics, for example, provided six pages to the subject, albeit in the form of modern experiments and mathematical analysis, largely as Michelson had done - as an experimental demonstration

S. F. JoHnSTON, "Making light work: practices and practitioners of light measurement," History of Science 34 (1996): 273-302.

${ }^{6}$ A. A. Michelson and E. W. Morley, "On a Method of making the Wave-length of Sodium Light the actual and practical Standard of Length," Philosophical Magazine 24, no. 5 (1887): 463-566.

${ }^{7}$ A. A. Michelson, "On the Application of Interference Methods to Spectroscopy Measurements -- II.," Philosophical Magazine 34, no. 5 (1892): 280-299.

A. A. Michelson, "On the Application of Interference-Methods to Spectroscopic Measurements -- I.," Philosophical Magazine 31, no. 5 (1891): 338-346.

8 A. A. Michelson, Light Waves and Their Uses (Chicago: Univ of Chicago, 1902).

A. A. Michelson, "Recent progress in spectroscopic methods," Nature 88 (1912): 362.

A. A. Michelson, Studies in Optics (Chicago: Univ of Chicago Press, 1927).

${ }^{9}$ P. DRUDE, The Theory of Optics (New York: Dover reprint, 1902).

${ }^{10}$ E. F. Nichols and J. D. TeAR, "Short Electric Waves," Physical Review 21 (1923): 587-605; H. RuBENS and H. Hollnagel, "Measurements in the Extreme Infra-Red Spectrum," Philosophical Magazine 19 (1910): 761-782.

H. RuBens and O. Von BAEyer, "On Extremely Long Waves, emitted by the Quartz Mercury Lamp," Philosophical Magazine 21 (1911): 689-695; H. RuBENS and R. W. Wood, "Focal Isolation of Long HeatWaves," Philosophical Magazine 21 (1911): 249-261.

${ }^{11}$ F. A. JENKINS and H. E. White, Fundamentals of Optics (New York: McGraw-Hill, 1937); WoOD, Physical Optics .

${ }^{12}$ See S. F. Johnston, Fourier Transform Infrared: A Constantly Evolving Technology (Chichester: Ellis Horwood, 1991). 
showing considerable promise but few results, while Geometrical and Physical Optics discussed interferential spectroscopy as an introduction to the now-established method of FTS. ${ }^{13}$ By the later decades of the century, texts such as Optics 0 recast the subject as part of a formalised treatment of optical coherence, an approach that has since become de rigeur for modern optics. Longer biographical works on Michelson, on the other hand, neglect or mis-state his spectroscopic research. ${ }^{14}$. Thus the interest in interferential spectroscopy vacillated as it was repeatedly recategorised and re-evaluated in relation to other techniques.

The rise of Fourier transform spectroscopy during the 1960s, and its dominance from the 1980s as the most common form of optical spectroscopy, has particularly clouded the historical evaluation of Michelson's spectroscopic work. Practitioners of FTS - reliant on variants of Michelson's interferometer - challenged 'conventional' dispersive spectroscopy based on prisms and diffraction gratings. ${ }^{15}$ One tactic in this competition was to marshal historical evidence to portray FTS as a technique of noble lineage rather than as an immature and ill-founded method. Consequently practitioners' historical excursions tend towards a whiggish treatment that stresses conceptual origins and fundamental technical elements that were revived and consolidated in their times. They portrayed the rise of FTS as historically inevitable and facilitated by the post-war appearance of computers, mathematical formalism and the extension of spectroscopic research into the far infrared.

The first such genealogy of Fourier transform spectroscopy appeared in the mid 1960s. In it, the author portrayed Michelson's research as the direct antecedent of FTS, despite its very different motivations and scientific context. ${ }^{16}$ He reconstructed interferential spectroscopy as a conceptually sound but technically-handicapped FTS; thus: "since Michelson had no computer available for taking Fourier transforms, he invented a harmonic synthesizer...". ${ }^{17}$ Capsule histories in textbooks have reinforced this simplified genealogy; the first general text on FTS cited Michelson's 'rightful claim to have realized and illuminated the basic concepts of Fourier transform spectroscopy' and that he 'could have developed the field some 90 years ago if the rest of the prerequisite technology had not been so far behind'. ${ }^{18}$

Pierre Connes, a key figure in the expansion of FTS during the 1960s, has written more careful and nuanced histories of the work of Michelson and his contemporaries. ${ }^{19}$ These remain, however, internalistic accounts that address merely the technical questions of why Michelson's interferential spectroscopy did not immediately blossom into what was to become FTS, representing the case as one of missed opportunities. Connes, like Bell, concludes that Michelson and his contemporaries could together have generated a technically workable form of spectroscopy based on interference techniques at the turn of the twentieth century. He says little, however, about the influence of social context. Fin de siècle discovery and application are treated simplistically, despite the strong counter-evidence provided by the subsequent emergence of FTS in his own time - and motivating its ardent 1960s histories. Given this skewed and tendentious historiography, is there in fact an historical question to answer at all?

13 R. S. Longhurst, Geometrical and Physical Optics (London: Longman Group, 1973); J. STRONG, Concepts of Classical Optics (San Francisco: W. H. Freeman, 1958).

${ }^{14}$ N. A. o. S. AnON., "Albert A. Michelson," Biographical Memoirs 19, no. 4 (1938); D. M. LivingSTON, The Master of Light: A Biography of Albert A. Michelson (New York: Charles Scribners, 1973); L. S. SwENSON JR, The Ethereal Aether: A History of the Michelson-Morley-Miller Aether-Drift Experiments, 1880-1930 (Austin: University of Texas Press, 1972).

15 On the mid-twentieth century technical and social competition between FTS and dispersive spectroscopy, see S. F. Johnston, "In search of space: Fourier spectroscopy 1950-1970," in Instrumentation: Between Science, State and Industry, ed. B. Joerges and T. Shinn, Sociology of the Sciences Yearbook (Dordrecht: Kluwer Academic, 2000), 121-141.

16 FTS was then (mid 1960s) limited to the far infrared portion of the optical spectrum and to weak, broad-band absorption spectra. The FTS technique was promoted initially for its superior light grasp (étendue) rather than for having resolution potentially better than dispersive instruments. Interferential spectroscopy, on the other hand, had been employed to study narrow-band visual-spectrum emission sources at extremely high spectral resolution.

${ }^{17}$ E. V. LOEWENSTEIN, "The history and current status of Fourier transform spectroscopy," Applied Optics 5 (1966): 845-854, quotation p 846.

18 R. J. BeLL, Introductory Fourier Transform Spectroscopy (New York: Academic Press, 1972), p. 17.

${ }^{19}$ P. CONNES, "Early history of Fourier transform spectroscopy," Infrared Physics 24 (1984): 69-93.

P. ConNes et al., "Heinrich Rubens, Herbert Hollnagel, and Fourier transform spectroscopy," Optics News, no. March (1988): 6-14. 
The present paper reforms and broadens the questions, ignoring the subsequent development of FTS and the transformation of the 'failure' of interferential spectroscopy into a remarkable 'success'. How did Michelson's interests in spectroscopy develop? What research goals did he have regarding spectroscopy? How seriously did he promote his spectroscopic achievements? And to what extent were they, in fact, seen as 'spectroscopic'? In order to address these questions, further details of Michelson's work are needed.

\section{The technology and culture of interference research}

The most fertile research theme of Michelson's career was the refinement of precision optical measurement. His first publications concerned the speed of light, a measurement that he made with considerably better precision than did his near-contemporaries, Hippolyte Fizeau (1819-1896) and Leon Foucault (1819-1868). Michelson's oft-cited statement that 'our future discoveries must be looked for in the sixth decimal place' seems to support this rationale. ${ }^{20}$ In fact, though, Michelson was alluding to the need for high precision to seek new, more subtle phenomena (and indeed was voicing this scientific goal to rationalise his strong attraction to the development of precision experimental devices themselves). His work of the 1890s focused on this approach by adapting and applying his interferometer to a series of new problems.

The second and nearly as consuming theme was the study and technical exploitation of interference phenomena, especially by applying his eponymous interferometer. ${ }^{21}$ Michelson invented his interferometer in 1880 to measure the absolute velocity of the earth through space (he later revised this goal to measuring the drift between the aether and the earth - a considerably less ambitious intellectual objective, but identically demanding in instrumental terms).

The Michelson interferometer bears very little resemblance to a nineteenth-century spectrometer. It carries no angular scale for measuring the deviation of spectral rays by a prism or diffraction grating. Indeed, it has no dispersive element at all. It employs no slits and no optical elements other than two mirrors and one half-silvered mirror. Michelson, in fact, referred to it for over a decade as an 'interferential refractometer', devising it to detect the presumed subtle differences in delay in the propagation of waves of light parallel and perpendicular to motion with respect to the aether. The configuration of the interferometer was simple, if unintuitive: a beam of light striking the semi-silvered mirror was divided into two components, one transmitted and one reflected. The two beams each travelled down separate 'arms' to mirrors, which reflected them directly back, and recombined at the semisilvered mirror to be reflected to an observer's eye. The divided beams interfered with each other when they recombined. If there were a difference in the optical path lengths of the two arms of the interferometer, the returning waves would differ in phase, and would combine to make a brighter or dimmer beam of outgoing light. ${ }^{22}$ If there were any variation in angle between the rays making up the recombined beams, the observer would see 'fringes' of interference, appearing as dark and light bands or rings. ${ }^{23}$ For the interferometer, then, the fundamental observables were optical path difference and a corresponding fringe variation.

Its technical origins, design and purpose owe much to other contemporary interferometers, such as those of Augustin Fresnel and Fizeau. Indeed, the style and subject of most of Michelson's subsequent research was modelled closely on the expanding and largely French-dominated scientific sub-culture later denoted 'interferometry'. For Fizeau, Foucault and Michelson, high-precision experiments to measure interference phenomena and the speed of light in various media were central to confirming and exploiting the wave theory of light. Michelson's early work sought to refine and extend this French research. Michelson's studies mapped neatly onto those of Fizeau and Foucault: determination of the velocity of light in air (Fizeau, 1849; Foucault, 1850; Cornu, 1875; Michelson, 1878); velocity of light in moving water

${ }^{20}$ A. A. MicheLson, Address, Dedication ceremony for Ryerson Physical Laboratory, University of Chicago..

21 The instrument went by a variety of names over the following two decades. The name 'interferometer' dates from the mid 1890s in Michelson's writings; 'interferometry’ was not in common use among optical scientists until at a decade later.

22 The difference in optical path length was expected to be produced by motion of the earth through the aether. It could, of course, also be produced for other experimental purposes by adjusting the length of one arm by moving its mirror.

23 An entrance collimator, as in a conventional prism spectroscope, would yield a collimated output beam and a visual field of either uniform intensity or, if the two arm mirrors were not exactly normal to their beams, a field crossed by linear fringes. 
(Fizeau, 1859; Michelson \& Morley, 1886) and, determination of wavelength by counting interference fringes (Fizeau, 1862; Michelson, 1881).

Thus the context and culture of Michelson's researches, at least up to 1890, owed much to French undulatory studies and little to the equally expanding, but distinct, subject of spectroscopy. ${ }^{24}$

\section{From metrology to spectroscopy}

The trajectory of their studies of optical interference carried both Fizeau and Michelson towards spectroscopic research. In the most trivial sense, wavelength was an obvious nuisance for interferometry. Because 'white' light is composed of a spectrum of wavelengths, the interference fringes produced by different colours in an interferometer have different length scales. When the arms of the Michelson interferometer have precisely the same length, the waves of all wavelengths passing through it are in step and yield a bright output beam. When the lengths of the arms are unequal, however, the interference patterns of the various wavelengths fall out of step, and average out to a colourless grey. The fringes disappear, and the interferometer reveals nothing to measure. Hence the detection of large path differences (i.e. more than a few wavelengths) demanded monochromatic light. ${ }^{25}$ The study of monochromaticity and interferometry went hand-in-hand.

Fizeau realised that he could determine the absolute wavelength of light by counting fringes as the path length in his interferometer was changed. When the optical path difference between the separated beams increased by one wavelength, the light leaving the interferometer went through a cycle, e.g. from a maximum of intensity through a minimum and back to a maximum. Measuring wavelength became as straightforward as counting dark or bright fringes. By relating optical interference to physical distance in this fashion, highly precise metrology became possible. ${ }^{26}$ Michelson's innovation was to employ his aether-detecting 'refractometer' in just such a way, by the addition of a mechanism for adjusting the optical path length while counting fringes. ${ }^{27}$ This demanded a calibrated and high-precision screw thread to alter the path length of one of the two optical beams in the interferometer. The technology was readily available in the form of a micrometer-driven carriage to support the mirror of one arm.

In 1862 Fizeau had been able to infer the wavelength separation of a spectral doublet with his interferometer. When the two wavelengths were in step (in phase at zero-path difference of the interferometer) the fringes were of high contrast; when the wavelengths were exactly out of phase, however, the contrast of the fringes - the difference between their maximum and minimum intensities fell to zero. ${ }^{28}$ Using the sodium $D$ line, he counted some 500 fringes between the high- and low-contrast regions of path difference - in effect, measuring the interval of optical 'beats' visualised by the interferometer - and thus inferred their relative spectral separation $(\Delta \lambda / \lambda)$ of 1 part in 1000 .

24 Michelson nevertheless had some direct connection with spectroscopists. During his European study in 1881-2, he studied spectroscopy in the classes of Robert Bunsen; see LIVINGSTON, The Master of Light .

25 Demonstrations of optical interference before Michelson generally employed near-symmetrical arrangements of the two optical paths (e.g. Young's double-slit; Lloyd's mirror; Fresnel's mirrors; Billet's split lens; on the variety of two-beam interference experiments, see JENKINS and WHITE, Fundamentals of Optics , Chap 13). Because of the geometry of these configurations, the interference of non-monochromatic light at large path differences (so-called "high interference") was not widely appreciated. Michelson, in attempting to demonstrate his new interferometer to Marie Alfred Cornu in 1881, was chastened to discover how difficult fringes were to obtain when using 'white' light; (LIVINGSTON, The Master of Light ). For his aether drift experiments, Michelson wanted interferometer arms of precisely equal length, for which wavelength has little effect on the observed fringes. Thus the interferometer, during the 1880s, was devoid of spectroscopic significance for Michelson.

${ }^{26}$ While this was the most precise form of optical metrology proposed until then, it was not the first. In the previous decade, two methods based on the dispersion of light by diffraction gratings had been published: see PEIRCE, Nature 22 (1879): 99 and Brown, "On the Absolute Wave-Lengths of Light," American Journal of Science 33 (1887): 167. Fizeau's method was, however, more direct in relating the wavelength of light to a directly measurable physical quantity, i.e. the movement of a mirror on a calibrated thread.

${ }^{27}$ MiCheLsON and MoRLEy, "On a Method of making the Wave-length of Sodium Light the actual and practical Standard of Length,",: 463-566.

${ }^{28}$ More accurately, the fringe contrast fell to zero only if the two spectral lines had equal intensity and an infinitely narrow, or at least equal, spectral distribution. 
This disappearance of fringes provided spectral information, but was a complication for using the interferometer as a metrological instrument. Michelson noted this 'slight difficulty' in his paper proposing the sodium emission line as a standard of length. He suggested, as a solution, 'counting through' the regions of indistinct fringes by employing intermediate standards. As an aside in the final paragraph of the paper, he noted that emission lines of other elements - specifically thallium, lithium and hydrogen - 'gave interference up to fifty to one hundred thousand wave-lengths, and so could be calibrated similarly and 'used as checks on the determination with sodium'. The spectroscopic implications of the observations were clearly secondary for Michelson:

It may be noted, that in case of the red hydrogen-line, the interference phenomena disappeared at about 15,000 wave-lengths, and again at about 45,000 wave-lengths; so that the red hydrogen-line must be a double line with the components about one sixtieth as distant as the sodium-lines. ${ }^{29}$

The scaled-down and pathlength-adjustable form of the 'interferential refractometer' had been reconceived by Michelson as a highly precise comparator. ${ }^{30}$ This elegant metrological instrument nevertheless had an uneasy relationship with its subject. To serve in generating a reliable standard of length, the instrument required highly monochromatic spectral emissions. It is notable that Michelson made relatively little of this spectroscopic connection, and saw the results with other elements as straightforward analogies of the sodium spectrum. The 'problem' with hydrogen, he suggested, was that it was a close spectral doublet.

During the following six years, Michelson developed the technique of optical interference counting and calibration of intermediate physical standards. Despite his seeming half-realisation in the 1887 paper that the 'refractometer' had spectroscopic applications, Michelson's first explicit publication on spectroscopy identified a different source for his ideas: his interference measurements on stars. ${ }^{31} \mathrm{He}$ had, since 1890, been applying interference techniques to astronomical objects beyond the resolution of large telescopes. He found that, by interposing a double aperture in front of the telescope so that it received light from two mirrors having an adjustable separation - a device he later called a 'stellar interferometer' he was able observe interference fringes from the astronomical source of light. The contrast, or what he called the 'visibility', of these interference fringes varied as the mirror separation was changed. Michelson demonstrated that the variation in visibility with separation, or 'visibility curve', was related to the angular diameter of the star. At the same time, he broached one of the criticisms later to dog his research: the question of indirect measurement and inference:

As ordinarily understood, this increase of "accuracy" would be at the cost of "definition" (which in this sense is practically zero); but if by "definition" we mean, not the closeness of the resemblance of the image to the object, but the accuracy with which the form may be inferred, then definition and accuracy are increased in about the same proportion. ${ }^{32}$

Having made this connection between the 'visibility curve' and the angular size of a light source for his stellar interferometer, Michelson made the now straightforward conceptual extension to the analogous case of 'visibility curves' and 'spectral size' of light sources with his laboratory interferometer. At the centre of this realisation was a new way of looking at observation itself : the acceptance of the need to rely on inference, and a special meaning for visual definition.

Now referring to his instrument as a 'wave-comparer', Michelson argued the complement of his 1887 description, proposing the instrument to investigate the characteristics of 'monochromatic' light sources:

${ }^{29}$ Michelson and Morley, "On a Method of making the Wave-length of Sodium Light the actual and practical Standard of Length,",: 463-566, p 466.

30 Based on one of Michelson's papers (A. A. Michelson, "Measurement by light waves," American Journal of Science 39 (1890): 115-121), Richard Staley suggests that Michelson was categorising his new instrument in a systematic relationship to telescopes, microscopes and spectrometers by this stage; see R. STALEY, "Michelson's interferometer: instrument or experiment?," in Instrument -- Experiment: Historische Studien, ed. Christoph Meinel (Berlin: Verlag fur Geschichte der Naturwiss. und der Technik, 2000), 193-200. This interpretation seems to downplay Michelson's rather tentative understandings and investigations of the early 1890s.

31 The application of interferometry for measuring the diameter of stars had been proposed by Fizeau in 1868.

32 A. A. MicheLson, "Visibility of Interference-Fringes in the Focus of a Telescope," Philosophical Magazine 31, no. 5 (1891): 256-259, p. 256. 
The red hydrogen-line is a very close double; and the same is also true for the green thallium-line. Another instance of the class of problems which the method may help to solve is the effect of the velocity of the vibrating molecule in the line of sight in broadening the spectral lines. ${ }^{33}$

For the first time Michelson acknowledged in print that his instrument could be used to infer the optical spectrum at very high resolution. He acknowledged that spectral emissions were not merely combinations of infinitely narrow 'lines', but were in fact broadened by Doppler shifts (as he noted in popular lectures, 'these "lines" are not such in a mathematical sense, but have an appreciable width and a varying distribution of light'. ${ }^{34}$ His theoretical connections were no longer centred on undulatory theory. Instead, he sought to confirm theoretical derivations concerning the intrinsic shape of spectral lines, and their broadening with temperature and pressure.. In this first spectroscopic paper, Michelson devoted considerable space to deriving the mathematical relationship between spectral line shape and visible curves. Importantly, Michelson cited the work of Lord Rayleigh - later to become his most responsive commentator - on these matters. These theoretical cases were mathematically tractable but experimentally simplistic, generally considering a spectral singlet or doublet as a rectangular, triangular, sinusoidal or exponential line shape. Encouragingly, the derivation demonstrated that it should be possible, with his wave-comparer, to determine spectral line-widths, extremely close doublet separations and even lineshapes - all spectral characteristics being far beyond conventional spectroscopic methods, and having important implications for inferring the temperature of light sources.

In a companion experimental paper the following year, Michelson illustrated his interferometer again, which had now reverted to the name 'refractometer'. The latest version bore a somewhat closer resemblance to a dispersive spectrometer: the input side included a collimator, and the output side incorporated a telescope. ${ }^{35}$ Michelson provided measured visibility curves for spectrally-isolated emission lines of hydrogen, oxygen, sodium, zinc, mercury, cadmium and thallium, and showed how they varied with temperature and pressure of the luminous gases. He used these observations for two purposes: first, to estimate the actual spectral profiles of these emission lines and, second, to estimate experimental values assuming the kinetic theory of gases. Both suggested 'remarkable agreement' with expectations, and enormous potential for this unparalleled spectroscopic technique. In fact, Michelson suggested that the resolution possible with what he now dubbed Light-Wave Analysis was at least an order of magnitude greater than the best grating spectrometers, and ultimately boundless: 'indeed, if the width of the lines themselves be less than their distance apart, there can be no limit'. ${ }^{36}$

There Michelson left the technique. He published no extensions to his method for some five years, focusing instead on metrological applications of interference techniques. However, when Pieter Zeeman announced his discovery that magnetic fields widened the spectral emission lines of sodium, ${ }^{37}$ Michelson quickly applied his method of light-wave analysis to this special case. He examined individual emission lines of sodium, cadmium and other elements. He was able to infer from the changes in the visibility curve that the lines were doubled and tripled. Subsequent investigations showed the lines to be split in more complex, but symmetrical ways. ${ }^{38}$ Unlike his 1892 investigations, Michelson now faced spectral profiles consisting of as many as a dozen spectral lines. His task was to relate the measured visibility curves to the unobservable fine structure of the emission lines he studied. His approach had been to surmise a spectral distribution, and then to calculate, by hand, the shape of visibility curve that this collection of wavelengths would produce. This was a matter of adding up sine waves corresponding to the surmised frequencies in the source. Thus a spectral doublet would consist of two sine waves of slightly different frequency, and would generate the 'beats' observed in the experimentally observed fringes. In

33 Michelson, "On the Application of Interference-Methods to Spectroscopic Measurements -- I.,",: 338-346, p 338.

34 Michelson, Light Waves and Their Uses , p 83.

35 While the telescope in a spectrometer usually magnifies the spectrum to make angular measurement more precise, the telescope in the Michelson interferometer simply provides a uniform visual field from a fixed perspective, which is useful in improving photometric precision.

${ }^{36}$ MicheLson, "On the Application of Interference Methods to Spectroscopy Measurements -- II.,",: 280-299, p 297.

${ }^{37}$ P. ZEEmAn, "The Effect of Magnetisation on the Nature of Light Emitted by a Substance," Nature 55 (1897): 347.

38 MicheLson, Light Waves and Their Uses , pp 107-119. 
order to consider spectral lines of finite width, however, Michelson and his assistant, Samuel W. Stratton, devised a mechanical device to do the summation of sine waves for him. This 'harmonic analyser' (actually, a harmonic synthesiser, since it summed up frequencies to yield an approximation of the observed fringe visibility pattern) was applied to the Zeeman effect observations. ${ }^{39}$ The automation of this time-consuming part of interferential spectroscopy reduced the drudgery considerably. ${ }^{40}$

Thus, extending the resolution of dispersive spectroscopy by at least an order of magnitude, and revealing new spectroscopic phenomena beyond theoretical explanation, Michelson would appear to have vindicated his unconventional technique. Why, then, was it relegated to footnotes in subsequent histories of spectroscopy?

\section{Problems with interferential spectroscopy}

Several discrete factors can be identified for the social failure of interferential spectroscopy. The most important of these is probably Michelson's own misgivings about the method. Certain problems of practice, analysis and confidence underlie these misgivings. So, too, do criticisms from better theorists. More subtly still, the norms and practices of the culture of spectroscopy and photometry had a significant influence on the reception of Michelson's method by spectroscopic practitioners. Michelson was unsatisfactorily straddling cultures of spectroscopy, metrology and interference optics.

\section{Michelson's misgivings}

By 1891, when he first described his form of Light-Wave Analysis, Michelson had developed a reputation over some 13 years as a meticulous experimenter in high-precision optics. His own evaluation of the strengths and limitations of his technique therefore carried considerable weight. As noted by Robert W. Wood, another renowned American optical experimentalist, 'Michelson's results were due to his great skill... which resulted from his long experience and familiarity with his instrument'. ${ }^{41}$

Michelson never vaunted light-wave analysis as a replacement to existing techniques. In a lowkey and understated style, he merely portrayed it as an adjunct to other methods of acquiring knowledge. Indeed, having only gradually envisaged the possibilities of his interferometer, he seemed unclear in all his writings concerning the deeper implications and limitations of the technique, and diffident about investigating them further. This lack of certainty and commitment in his published writings contrasts strongly with his descriptions of the aether-drift experiment, for example. By 1896, commenting on interferential spectroscopy as a means of studying the Zeeman effect, he seemed aware of its problems for spectroscopy as typically practised:

The examination of spectral lines by means of the interferometer, while in some respects ideally perfect, is still objectionable for several reasons. In particular, it requires a very long time to make a set of observations, and we can examine only one line at a time. The method of observation requires us to stop at each turn of the screw, and not the visibility of the fringes at each stopping-place. During the comparatively long time which it takes to do this the character of the radiations themselves may change.

Besides, we have the trouble of translating our visibility curves into distribution curves. Hence it is rather easy for errors to creep in. ${ }^{42}$

39 A. A. Michelson, "A New Harmonic Analyser," Philosophical Magazine 45, no. 5 (1898): 85-91.

40 This mechanical device, extended from a 20-element prototype, could sum 80 spectral elements and thus generate a reasonably precise synthetic visibility curve from a surmised spectrum. The inference of the actual spectrum was a trial and error affair, however. Copies of the analyser are housed at the Smithsonian Institution in Washington and the Science Museum in London, where it is described as an 'integrator'. While Michelson noted that 'the machine is capable not only of summing up any given trigonometric series, but can also perform the inverse process of finding for any given function the coefficients of the corresponding Fourier series' (Ibid.), it seems doubtful that he regularly used it in this way to calculate the spectrum from his visibility curve. Despite his strong implication in popular lectures (e.g. MicheLSON, Light Waves and Their Uses , p 73), fringe visibility did not translate into a unique spectrum; the harmonic analyser adjustments for this inverse problem were very sensitive to the experimenter's assumptions about the unobservable spectrum.

${ }^{41}$ Wood, Physical Optics , p 411.

42 Michelson, Light Waves and Their Uses , pp 118-119. 


\title{
The 'translation' problem
}

The primary problem in Michelson's opinion, despite his remarks about lengthy observations, was that of translation. There was an unsettling difficulty in inferring the spectrum of the light source from its variation in fringe contrast, or the visibility curve, produced by the interferometer. Concerns about the translation from visibility curve to spectral distribution appear to have been articulated in step with his growing awareness of the latent power of interferential spectroscopy.

Michelson entered into correspondence with Lord Rayleigh, seeking a confirmation of 'the definitiveness with which the spectral line $\phi(x)$ can be deduced from the "visibility curve". Rayleigh observed that the two distributions were related by Fourier's theorem, and that there was an inherent ambiguity between what Michelson was able to measure and the spectrum itself. According to the theorem, the spectrum $\phi(x)$ was a function of the optical path difference $x$, and was a combination of cosine terms $(\mathrm{C})$ and sinusoidal terms $(\mathrm{S})$. Rayleigh noted that the visibility curve provided not $\mathrm{C}$ and $\mathrm{S}$ but only $\mathrm{C}^{2}+\mathrm{S}^{2}$, and so

\begin{abstract}
we must conclude that in general an indefinite variety of structures is consistent with a visibility-curve given in all its parts... In order to determine both $\mathrm{C}$ and $\mathrm{S}$, observations would have to be made not only upon the visibility, but also upon the situation of the bands [that is, on the position of interference fringes]. You remark that "it is theoretically possible by this means to determine, in case of an unequal double, or a line unsymmetrically broadened, whether the brighter side is towards the blue or the red end of the spectrum". But I suppose that a complete determination of both $\mathrm{C}$ and $\mathrm{S}$, though theoretically possible, would be an extremely difficult task.
\end{abstract}

Rayleigh concluded that 'in one case we have to deal with amplitudes and in the other with intensities. ${ }^{43}$ Thus, in a succinct letter supported by mathematical derivations, Rayleigh indicated that interferential spectroscopy could be a satisfactory means of inferring spectra in certain special cases, but not as a general solution. It measured the relative intensity, or contrast, of interference fringes, rather than measuring their position and amplitude, and it did so over a limited range of optical path difference. This was adequate when examining narrow spectral lines, or symmetrical combinations of several spectral lines, but it would give ambiguous results in more complicated spectral profiles. The Zeeman effect was fortuitously an almost ideal case for interferential spectroscopy - a symmetrical spectrum, consisting of a handful of spectral components capable of Fourier analysis by hand calculation, and timed perfectly for Michelson's still active interest in the method.

Michelson was more or less aware of most of Rayleigh's criticisms. He had earlier proposed a means of measuring the position as well as the intensity of his fringes, thereby providing enough information to decide about non-symmetrical spectral distributions. ${ }^{44}$ There is no evidence, however, that he ever attempted the measurements, or consequent analysis, in practice. Indeed, he was quick to propose conventional dispersive spectroscopy as the final arbiter in decision-making: 'Doubtless in many cases where the components are not too close, the grating will give the information necessary for the investigator to select the proper combination'. ${ }^{45}$ Interferential spectroscopy was conceptualised as an adjunct method to dispersive techniques, in effect magnifying the resolving power of gratings over narrow ranges.

Thus, while accepting this partial vindication for interferential spectroscopy - at least enough to apply the method to the study of the Zeeman effect - Michelson was continually, and publicly, troubled by its inherent indirectness, ambiguous inference and time-consuming analysis.

43 L. RAYleigh, "On the Interference Bands of Approximately Homogeneous Light; in a Letter to Prof. A. Michelson," Philosophical Magazine 34, no. 6 (1892): 407-411, pp 407-8. Rayleigh returned to a more general treatment of Fourier analysis in physical two decades later L. RAYLEIGH, "Remarks concerning Fourier's Theorem as applied to Physical Problems," Philosophical Magazine 24, no. 6 (1912): 864-869.

44 Michelson, "On the Application of Interference-Methods to Spectroscopic Measurements -- I.,",: 338-346, pp 344-5.

45 Michelson, "On the Application of Interference Methods to Spectroscopy Measurements -- II.,",: 280-299, p 297. 


\section{Substituting the echelon for the interferometer}

Michelson suggested that the application of the interferometer to the investigation of the Zeeman effect was a motivation in seeking something more akin to conventional dispersive spectroscopy:

On account of these limitations of the interferometer method, attention was directed to something which should be more expeditious, and the most promising method of attack seemed to be to try to improve the ordinary diffraction grating. ${ }^{46}$

He appears to have written nothing further on the genesis of the echelon, first described in 1898. What was the echelon? Michelson described it as a reconceived diffraction grating. He noted that 'conventional' gratings had well-known problems. ${ }^{47}$ Light was dispersed into several spectral 'orders', which overlapped increasingly at higher orders. Moreover, the difficulty of their production yielded spectra having uncontrollable intensity in the desired higher 'order'. Michelson reconceived the grating specifically for high-resolution use. Instead of relying on fine, scribed parallel lines, he constructed a coarse transmission grating from stacked glass plates of accurately equal thickness. These geometrically identical elements dispersed all the energy into a single spectral order. The stacked plates, in some cases more than $2 \mathrm{~cm}$ thick, yielded very high-order interference and thus a spectral dispersion much higher than a conventional grating. To avoid the problem of overlapping orders, Michelson filtered the incoming light with a prism monochromator. ${ }^{48}$

Thus the echelon spectroscope was an extremely narrow-band device resembling a two-stage conventional spectrometer. It was used in the conventional way; there was no observation of interference fringes to be made, and no 'translation' between visibility curves and spectral distributions. Michelson portrayed his interferential spectroscopy as a technique having 'serious drawbacks', and the echelon as a more natural genealogical link with spectroscopy and yet broadly comparable to the interference method in power:

A new instrument was devised, the echelon spectroscope, which has all the advantages of the grating spectroscope, together with a resolving power many times as great. With the aid of this instrument all the preceding deductions have been amply verified and a number of new and interesting facts added to the store of our knowledge.... ${ }^{49}$

It was, indeed, a particularly well-made diffraction grating, like several successors developed by other researchers. ${ }^{50}$ Nevertheless, Michelson had not wholly abandoned interferential spectroscopy for conventional dispersive techniques. His principal innovation was in describing the new optical element in terms of optical interference, and in making explicit the connection between interference phenomena and high-resolution spectroscopy. Interferential spectroscopy prepared spectroscopists for a new domain of investigation - high resolution, narrow band spectroscopic research. At the same time, he offered the echelon as a direct alternative to the interferometer, applying both to a practical problem of considerable contemporary interest (the Zeeman effect). The direct and timely comparison of these two instruments was decisive. Given Michelson's status as a precision experimenter, his rejection of interferential spectroscopy as an inferior technique was sufficient reason for many practitioners to ignore it.

\footnotetext{
${ }^{46}$ Michelson, Light Waves and Their Uses , pp 118-119.

47 The two principal varieties were wire-wound coarse gratings, and finer gratings constructed from scribed parallel lines on glass.

48 Michelson, Light Waves and Their Uses , pp 119-126.

${ }^{49}$ Ibid.,, , p 126.

50 Unlike for interferential spectroscopy, other investigators quickly responded to the promise of the new echelon plate. The echelon was employed for high-resolution spectroscopy, and was joined by the Fabry-Pérot etalon (1899) (J. M. VAughan, The Fabry-Perot Interferometer: History, Theory, Practice and Applications (Bristol: Hilger, 1989)), Wood's echelette, subsequent echelles and the Lummer-Gehrcke plate. For an abbreviated discussion, see Michelson, Studies in Optics , pp 104-110. A more recent and generic discussion of these devices is given in LONGHURST, Geometrical and Physical Optics , pp 175-92, 204-5 and 276-81. Echelles, echelettes and echelons can all be categorised as different geometries of gratings all having a particularly welldefined groove angle, or blaze. It should be noted that Michelson himself attempted to improve upon manufactured diffraction gratings by developing a ruling engine to rival that of Henry Rowland.
} 


\section{Conflicting consistency: metrology versus photometry}

The timing and context of the inter-comparison were therefore important. There were, however, disjunctions inherent in interferential spectroscopy. The first was that fringe counting could yield exquisitely precise determinations of wavelengths, and yet Michelson employed his method of light wave analysis only to infer relative profiles of spectral emissions, usually devoid of a wavelength scale. A second incongruity was that of a high-precision measurement relying on delicate interference effects, but combined with mathematical ambiguity in the spectral distribution. Such an imbalance between observation and analysis was disquieting, but not uncommon. The study of blackbody radiation is a contemporaneous case in which painstaking measurements were only later combined with an adequate theoretical 'fit'. ${ }^{1}$ Yet the careful spectro-radiometric measurements that characterised blackbody experiments in the 1890s highlight another disjunction specific to Michelson's interferential spectroscopy: that between careful observations of interference metrology and the incomparably vague estimation of intensity. A close parallel hinting at contemporary dissatisfaction with visual methods is the case of Nrays. René-Prosper Blondlot, from 1903, was to be criticised for his reliance on the detection of brightness changes by eye. ${ }^{52}$

Michelson's spectroscopic work combined two measurement approaches in surprising ways. On the one hand, as we have seen, his experience in interference measurement had produced remarkable achievements of precision. On the other, he made only the crudest of attempts to judge the contrast of his interference patterns. Both were nonetheless important for a precise reconstruction of the spectrum. As Rayleigh had suggested, interferential spectroscopy needed more than Michelson had supplied. It required careful observation of the shape of interference fringes, i.e. of their position and intensity. Michelson observed position quite precisely - by counting fringes - but made little serious attempt to measure their brightness. He recorded merely the envelope of the fringe pattern, not its detailed point-bypoint variation.

The disparity between his observations - between the precise abscissa and the crude ordinate of his visibility plots - was profound for an experimenter whose reputation relied on unparalleled precision. Michelson was satisfied with visual estimates of brightness during a scientific period when they were being refined into a highly polished photometric technique. ${ }^{53}$ Visual photometric measurements, for applications ranging from stellar magnitude to standards of gas lighting, were being quantified with increasing precision. Yet Michelson was content with a considerably lower standard: 'It has been found that the simple eye-estimates of the distinctness of the fringes coincide fairly well with the results given by [the mathematical expression for visibility]', he noted. ${ }^{54}$ His assurances nevertheless belie his claims. In the companion experimental paper Michelson described a calibration of his visual estimates. ${ }^{55}$ He used a pair of Nicol prisms to adjust intensity according to Malus' law of crossed polarisers. By comparing his own estimates of fringe contrast to this reference, he reported systematic errors up to $16 \%$. This was distinctly poorer than contemporary norms; photometrists typically reported precision of $1 \%$ for careful visual observations of intensity. Michelson noted that the systematic error also increased

...with the refrangibility of the light used; it is greater when the field contains a large number of bands than when there are but few; it is greater while the visibility-curve is

${ }^{51}$ H. KANGRO, Early History of Planck's Radiation Law (London: Taylor \& Francis, 1976); T. S. KuHN, BlackBody Theory and the Quantum Discontinuity (Oxford: Oxford University Press, 1978).

52 The literature on N-rays is extensive; see, for example, C. GELAin and H. GEOFFroy, "À la poursuite des Rayons "N"," L'ingenieur des Industries Chimiques 1965, 7-12; R. T. LAGEMANN, "New Light on Old Rays: N rays," American Journal of Physics 45 (1977): 281-284; M. J. NYE, "N-rays: An episode in the history and psychology of science," Historical Studies of the Physical Sciences 11, no. 1 (1980): 125-156; G. F. STRADLING, "A resumé of the literature of the $\mathrm{N}$ rays, the $\mathrm{N}_{1}$ rays, the physiological rays and the heavy emission, with a bibliography," Journal of the Franklin Institute 164 (1907): 57-74, 113-130, 177-199. For its specific connection with 'inadequate photometry' and norms of measurement, see JoHnSTON, A History of Light and Colour Measurement, pp 58-64.

53 On the rise of quantitative visual photometry, see ibid., Chap. 3].

${ }^{54}$ Michelson, "On the Application of Interference-Methods to Spectroscopic Measurements -- I.,",: 338-346, p 346.

55 MicheLson, "On the Application of Interference Methods to Spectroscopy Measurements -- II.,",: 280-299. 
falling than when it is rising; ... finally, it varies on different occasions and with different observers. ${ }^{56}$

Michelson was less explicit about random errors in estimating intensity, but in describing the particularly complex visibility curve for the green line of mercury he was equally sanguine:

...we might imagine that another series of measurements would give quite a different curve. But I have actually made over one hundred such measurements, and each time obtained practically the same results, even to the minutest details of secondary waves. ${ }^{57}$

In later descriptions, Michelson emphasised the role of visual experience (much as the rapidly discredited Blondlot was to do a decade later), ${ }^{58}$ while minimising importance of photometric precision:

By the use of fringes which have a known visibility we can educate the eye in estimating visibility, or we may have these standard fringes before us for comparison at the time of observation, and may then determine when the two systems are of the same clearness; and when they are of the same clearness, we may say that the desired visibility is the same as that whose value is known from our formula. This is the more accurate method, and is the one which was finally adopted; but long before its adoption it was found that fairly accurate visibility curves could be obtained by merely agreeing to call the visibility 100 when it was perfect, 75 when it was good, and 50 when it was fair... As a matter of fact, however, it is not of the utmost importance to determine the visibility with great accuracy. We know that we can measure a minimum or a maximum independently of any scale, and these points are really important ones... even without any very great amount of experience in observation, we can get a very fair visibility curve, and from that a very fair conception of the nature of the spectrum of the particular source we are examining... 59

Incongruously, the experimenter distinguished for high-precision measurement seemed content with a 'fair conception' of the simple spectral sources he examined.

\section{Confronting cultures}

As illustrated above, Michelson sidled toward spectroscopy while pursuing other research interests. His papers display a growing awareness of the implications of interference methods in spectroscopy, but little concern for promoting them to spectroscopists.

Michelson contrasted his 'light-wave analysis' with 'ordinary spectrum analysis' ${ }^{60}$ The distinction between these two fields of research, and the instruments they employed, were distinct in his writings. However, not until his development of the echelon and the study of Zeeman spectra for limited intercomparison with light-wave analysis did he categorise both as effective tools for spectrum analysis.

The problem with intensity measurement hints at wider disjunctions between scientific cultures. As Swenson has observed, 'spectroscopy' and 'interferometry' coalesced into 'autonomous sciences in their own right... made possible but also made inevitable by the development of ... fecund instruments', a theme more recently rehearsed by Chen. ${ }^{61}$ Michelson had developed certain skills, neglected others, and largely side-stepped contemporary concerns in spectroscopic practice. Light-wave analysis demanded an intimate knowledge of optical interference - still a relatively unexplored phenomenon for most optical scientists, and unfamiliar to the majority of spectroscopists. Practical knowledge was crucial for this

56 Ibid., p 281. This appears to be the only published reference concerning other observers of fringe visibility. Morley had collaborated extensively on aether-drift observations, but this relied upon detecting deviation of the position of a fringe rather than judging changes in its contrast.

57 Michelson, Light Waves and Their Uses, p 80.

58 For a similar justification of the need for trained eyes in N-ray observations, see R.-P. BLONDLOT, The N-Rays, trans. J. Garcin (London: Longmans, Green, 1905), pp 82-3.

${ }^{59}$ MicheLson, Light Waves and Their Uses , pp 74-5.

60 Ibid., p 79.

61 Swenson JR, The Ethereal Aether: A History of the Michelson-Morley-Miller Aether-Drift Experiments, 18801930 , p 58; X. CHEN, Instrumental Traditions and Theories of Light: The Uses of Instruments in the Optical Revolution (Dordrecht: Kluwer, 2000). 
experimental activity: tacit knowledge about the precision required of mirror surfaces (some ten to fifty times flatter than for normal imaging); mechanical stability and motion controlled to small fractions of a wavelength; the damping of external vibrations to which the interferometer was exquisitely sensitive; and, optical alignment to arc-second tolerances.

By contrast, other forms of tacit knowledge relevant to spectroscopists had little importance for interferential spectroscopy. Angular measurement was not a part of Michelson's method. There were no precise slits to be constructed. Knowledge of prism dispersion or grating artefacts was irrelevant. Means of comparing two spectra - increasingly important for routine identification and for determining spectral shifts caused by velocity - were a technology alien to light-wave analysis. On the other hand, Michelson's method relied on visual observation - but observation of a style and precision unfamiliar to spectroscopists. Spectrum analysis with dispersive instruments, especially for astronomical spectroscopy, employed photographic recording of spectra. These records were very different from what Michelson produced. They were integrative rather than sequential, and so produced results more simply and less tediously. They could be examined by more than one person, helping to demonstrate validity and to consolidate trust. They could be analysed to carefully determine intensity variations. Most obviously, photographic spectra covered a very broad spectral range; the quantity of interest could be observed directly. ${ }^{62}$

The purposes of spectroscopy illustrate cultural collisions at a different scale. Conventional dispersive spectroscopy in the late $19^{\text {th }}$ century was concerned with chemical identification and limited quantitative analysis, and, for stellar light sources, the determination of relative velocity. Michelson's spectroscopy, on the other hand, focused on determining the fine structure of individual spectral lines - a type of information irrelevant to spectroscopists' immediate concerns.

\section{Contemporary reception}

Experimental techniques deemed inappropriate or intricate are frequently disregarded. So it was with Michelson's method. The only serious challenge, as noted by Staley, was mounted by Thomas Preston, a spectroscopist in Dublin. ${ }^{63}$ Preston argued that the anomalous Zeeman effect reported by Michelson was an instrumental artefact, and hinted that his reputation had dampened debate. ${ }^{64}$ Interferential spectroscopy was otherwise discussed by few contemporaries, with the notable exception of Lord Rayleigh as described above. Two other investigators, Heinrich Rubens and Ernest Fox Nichols, made limited use of it one to two decades later.

During 1910 and 1911, Rubens and collaborators employed another form of interferometer to infer the spectrum of infrared sources. ${ }^{65}$ Even for these investigators, the research context was considerably different from Michelson's. High spectral resolution was not on their agenda; they sought, instead, a sensitive means of measuring weak radiation:

In obviating such a diminution of energy, the interference method has a decided advantage over all spectrometric methods, where the use of a slit is unavoidable. A second advantage consists in the evasion of the diffraction grating which is so uneconomical of energy. ${ }^{66}$

Their observational technique was also distinctly different from Michelson's. Rather than observing the contrast of visible fringes, they employed a radiometer to measure the variation of intensity of the

${ }^{62}$ Here, again, Michelson again appears to be an incongruous transitionary figure in optical cultures. As Hentschel discusses (P. K. HENTSCHEL, "Spectroscopy or spectroscopies?," Nuncius 17 (2002)), spectroscopists during this period were often highly oriented toward a visual culture. Michelson, himself an accomplished artist and eloquent on the beauty of light and colour, sacrificed this source of information when developing interferential spectroscopy.

63 STALEY, "Travelling Light,", 37-65.

64 T. Preston, "The interferometer," Nature 59 (1899): 605

T. PRESTON, "Radiation phenomena in the magnetic field," Nature 59 (1899): 224-229.

65 Rubens and Hollnagel, "Measurements in the Extreme Infra-Red Spectrum,",: 761-782

RuBEns and Von BAEYeR, "On Extremely Long Waves, emitted by the Quartz Mercury Lamp,",: 689-695

RuBENS and WoOD, "Focal Isolation of Long Heat-Waves,",: 249-261.

${ }^{66}$ RuBENS and Hollnagel, "Measurements in the Extreme Infra-Red Spectrum,",: 761-782, p 761. 
(invisible) fringes of infrared radiation. The partition of research strategies according to spectral range had been a long-standing division, and one that segregated the cultures of radiometry and photometry. ${ }^{67}$ Thus Rubens and co-workers scarcely referred to Michelson's work and did not attempt a systematic method of 'translating' their observations into the spectra. Ignoring Rayleigh's critique and Michelson/Stratton harmonic analyser, they used Michelson's 1892 method of fitting presumed spectral lines to the observed fringe patterns:

It is possible to get an idea of the approximate form of the energy curve of the radiation isolated by the quartz lenses, by a trial and error method. ${ }^{68}$

A decade later, Nichols sought to measure the characteristics of radiation between Rubens' infrared wavelengths and the longer 'radio wave' range of the spectrum. Nichols employed another interferometer design equivalent to that of Michelson and Rubens, but further isolated the wavelengths of interest with a reflecting echelon. He employed this instrumental combination as Rubens had done, using an electrical receiver to measure the intensity of fringes at successive optical path differences. As with Michelson's 1892 method and Rubens' work, Nichols relied on a hand-calculated trial and error analysis to infer the spectrum from his fringe measurements. ${ }^{69}$ In later measurements, he relied more closely on echelons. ${ }^{70}$ Nichols, a close acquaintance of Michelson, did not mention the source of these experimental tools and analytical methods in his paper.

\section{Conclusions}

Michelson, largely alone, pursued his interferential spectroscopy actively for less than a decade. His research motivations during that period combined a changing mixture of undulatory theory, precision measurement, metrology and spectrum analysis. Instrumentation was central to all of these concerns, and Michelson repeatedly mutated his technology and his conceptualisations of it:

- 1880-1: an 'interferential refractometer' for detecting differences in light velocity in different directions, and having 'arms' of fixed and equal path-length.

- $\quad$ 1887: a 'refractometer' as a tool for optical metrology using the principle of fringe-counting, via the addition of a micrometer-adjusted optical path-length.

- $\quad$ 1891: a 'wave comparer' for analysing light waves, including the absolute wavelength and determination of the separation of spectral double lines.

- $\quad$ 1892: a 'refractometer' for inferring spectral profiles, reliant upon measured 'visibility curves' and inferring the spectrum by hand-calculation.

- $\quad$ 1897-1898: an 'interferometer' for investigating spectral profiles produced by the Zeeman effect, using experimental techniques unchanged from 1892.

- 1898: an 'interferometer' + 'harmonic analyser' for more systematic inference of spectral profiles.

- 1898 : invention of the 'echelon plate' for direct high-resolution spectroscopy, and its substitution for the 'method of light wave analysis'.

Over these two decades, the only constant feature was the interferometer and applications of optical interference. Hence the programme of exploiting a new experimental technique led him into new terrain. Michelson's version of high-resolution spectroscopy was a transient application, haltingly conceived and diffidently developed. The tentative foray into spectroscopic territory brought Michelson into confrontation with contrasting research methods and research goals. His definition of spectroscopy itself was unconventional: it was one of precise wavelength measurement and high-resolution but narrow-band spectral distributions. The tools and methods were equally original, based on arcane and delicate observations supported by arduous and unsatisfying mathematical transformations. He was a hesitant promoter, apologetic for the ambiguity of his methods which seemed to reveal hidden secrets imperfectly. The reasons for the demise of the method are thus varied, but they centre on Michelson as its chief advocate and denigrator. He was simultaneously an authority on high-resolution spectroscopy (and thus competent to judge the superiority of the echelon over the interferometer) and yet an awkward fit to the culture of spectroscopy (employing observational methods that were rapidly falling from fashion).

\footnotetext{
67 Johnston, A History of Light and Colour Measurement: Science in the Shadows , pp 24-6, 220-1, 230-3.

68 RuBENS and WoOD, "Focal Isolation of Long Heat-Waves,",: 249-261, p 254.

${ }^{69}$ NichOLs and TEAR, "Short Electric Waves," 587-605.

${ }^{70}$ LivingSTON, The Master of Light, p 298.
} 
Spectroscopists found high-resolution studies to be a fertile field, but only when employing the familiar technology of the echelon plate. The alien and temperamental interferometer, with its ambiguous analysis, left them unconvinced.

\section{References}

Anon., National Academy of Sciences. "Albert A. Michelson." Biographical Memoirs 19, no. 4 (1938). BeLl, RoberT JOHN. Introductory Fourier Transform Spectroscopy. New York: Academic Press, 1972.

Blondlot, RenÉ-Prosper. The N-Rays. Translated by J. Garcin. London: Longmans, Green, 1905. Brown. "On the Absolute Wave-Lengths of Light." American Journal of Science 33 (1887): 167.

CHEN, XIANG. Instrumental Traditions and Theories of Light: The Uses of Instruments in the Optical Revolution. Dordrecht: Kluwer, 2000.

CONNES, PIERRE. "Early history of Fourier transform spectroscopy." Infrared Physics 24 (1984): 69-93.

Connes, Pierre, Michael Smyth, Armand Hadni, and Xavier Gerbaux. "Heinrich Rubens, Herbert Hollnagel, and Fourier transform spectroscopy." Optics News, no. March (1988): 6-14.

Drude, Paul. The Theory of Optics. New York: Dover reprint, 1902.

Gelain, C., and H. GeOFFroy. "À la poursuite des Rayons "N"." L'ingenieur des Industries Chimiques 1965, 7-12.

HeNTSCHEL, P. KLAus. "Spectroscopy or spectroscopies?" Nuncius 17 (2002).

Jenkins, Francis A., and Harvey E. White. Fundamentals of Optics. New York: McGraw-Hill, 1937.

JoErges, BERNWARD, and TERry SHInN, eds. Instrumentation: Between Science, State and Industry. Dordrecht: Kluwer, 2000.

JOHNSTON, SEAN F. Fourier Transform Infrared: A Constantly Evolving Technology. Chichester: Ellis Horwood, 1991.

-A History of Light and Colour Measurement: Science in the Shadows. Bristol: Institute of Physics Publishing, 2001.

"In search of space: Fourier spectroscopy 1950-1970." In Instrumentation: Between Science, State and Industry, edited by Bernward Joerges and Terry Shinn, 121-141. Dordrecht: Kluwer Academic, 2000.

"Making light work: practices and practitioners of light measurement." History of Science 34 (1996): 273-302.

Kangro, Hans. Early History of Planck's Radiation Law. London: Taylor \& Francis, 1976.

KLEIN, MiLes V. Optics. New York: John Wiley, 1970.

Kunn, Thomas S. Black-Body Theory and the Quantum Discontinuity. Oxford: Oxford University Press, 1978.

Lagemann, Robert T. "New Light on Old Rays: N rays." American Journal of Physics 45 (1977): 281-284.

Livingston, Dorothy Michelson. The Master of Light: A Biography of Albert A. Michelson. New York: Charles Scribners, 1973.

LOEWENSTEIN, ERNEST V. "The history and current status of Fourier transform spectroscopy." Applied Optics 5 (1966): 845-854.

LONGHURST, R. S. Geometrical and Physical Optics. London: Longman Group, 1973.

Michelson, Albert A. Address, Dedication ceremony for Ryerson Physical Laboratory, University of Chicago. Chicago, 1894.

. Light W aves and Their Uses. Chicago: Univ of Chicago, 1902.

. "Measurement by light waves." American Journal of Science 39 (1890): 115-121.

"A New Harmonic Analyser." Philosophical Magazine 45, no. 5 (1898): 85-91.

. "On the Application of Interference Methods to Spectroscopy Measurements -- II." Philosophical Magazine 34, no. 5 (1892): 280-299.

"On the Application of Interference-Methods to Spectroscopic Measurements -- I." Philosophical Magazine 31, no. 5 (1891): 338-346.

"Recent progress in spectroscopic methods." Nature 88 (1912): 362.

Studies in Optics. Chicago: Univ of Chicago Press, 1927.

"Visibility of Interference-Fringes in the Focus of a Telescope." Philosophical Magazine 31, no. 5 (1891): 256-259.

Michelson, Albert A., and Edward W. Morley. "On a Method of making the Wave-length of Sodium Light the actual and practical Standard of Length." Philosophical Magazine 24, no. 5 (1887): 463-566.

Nichols, E. F. , and J. D. Tear. "Short Electric Waves." Physical Review 21 (1923): 587-605.

NyE, MARY JO. "N-rays: An episode in the history and psychology of science." Historical Studies of the Physical Sciences 11, no. 1 (1980): 125-156.

PEIRCE. Nature 22 (1879): 99. 
PReston, Thomas. "The interferometer." Nature 59 (1899): 605.

. "Radiation phenomena in the magnetic field." Nature 59 (1899): 224-229.

RAYLEIGH, LORD. "On the Interference Bands of Approximately Homogeneous Light; in a Letter to Prof. A. Michelson." Philosophical Magazine 34, no. 6 (1892): 407-411.

. "Remarks concerning Fourier's Theorem as applied to Physical Problems." Philosophical Magazine 24, no. 6 (1912): 864-869.

Rubens, H., and H. Hollnagel. "Measurements in the Extreme Infra-Red Spectrum." Philosophical Magazine 19 (1910): 761-782.

Rubens, H., and O. VON BAeYer. "On Extremely Long Waves, emitted by the Quartz Mercury Lamp." Philosophical Magazine 21 (1911): 689-695.

Rubens, H., and R. W. Wood. "Focal Isolation of Long Heat-Waves." Philosophical Magazine 21 (1911): 249-261.

STALEY, RICHARD. "Michelson's interferometer: instrument or experiment?" In Instrument -- Experiment: Historische Studien, edited by Christoph Meinel, 193-200. Berlin: Verlag fur Geschichte der Naturwiss. und der Technik, 2000.

"Travelling Light." In Natural Standards, 37-65: Max-Planck-Institut fur Wissenschaftsgeschichte, 2001.

STRADLING, GEORGE FlOWERs. "A resumé of the literature of the $\mathrm{N}$ rays, the $\mathrm{N}_{1}$ rays, the physiological rays and the heavy emission, with a bibliography." Journal of the Franklin Institute 164 (1907): 57-74, 113-130, 177-199.

Strong, John. Concepts of Classical Optics. San Francisco: W. H. Freeman, 1958.

Swenson JR, LOYD S. The Ethereal Aether: A History of the Michelson-Morley-Miller Aether-Drift Experiments, 1880-1930. Austin: University of Texas Press, 1972.

Vaughan, J. M. The Fabry-Perot Interferometer: History, Theory, Practice and Applications. Bristol: Hilger, 1989.

WoOD, R. W. Physical Optics. New York: MacMillan, 1923.

ZEeman, Pieter. "The Effect of Magnetisation on the Nature of Light Emitted by a Substance." Nature 55 (1897): 347. 